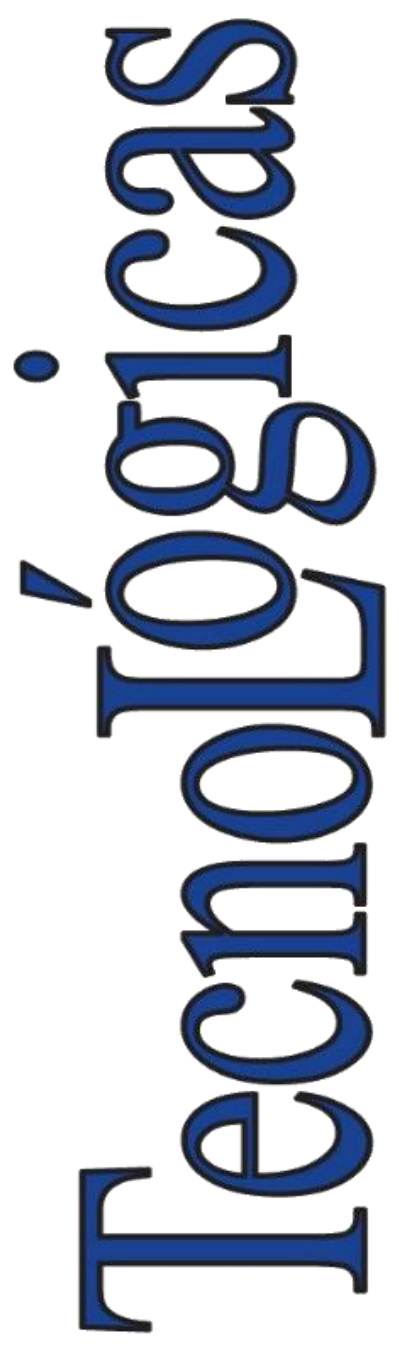

ISSN-p: 0123-7799

ISSN-e: $2256-5337$

Vol. 24, nro. 51, e1738, 2021

Recibido: 24 agosto 2020 Aceptado: 18 diciembre 2020 Disponible: 26 febrero 2021

CInstituto Tecnológico Metropolitano Este trabajo está licenciado bajo una Licencia Internacional Creative Commons Atribución (CC BY-NC-SA)

\section{Concentración de metales pesados en suelos agrícolas bajo diferentes sistemas de labranza}

\section{Concentration of Heavy Metals in Soils Under Different Tillage Systems}

\author{
(D) Betty Mendoza-Escalona ${ }^{1}$; \\ (D) Duilio Torres-Rodríguez; \\ (D) Lué Merú Marcó3; \\ (iD) Carlos Gómez ${ }^{4}$; \\ iD Marisela Estanga-Barrios; \\ (D) Yelitza García-Orellana ${ }^{6}$
}

1 Universidad Rey Juan Carlos, Madrid, España, bmendoza2803@gmail.com

2 Universidad Centroccidental Lisandro Alvarado, Cabudare, Venezuela, duiliotorres@ucla.edu.ve

${ }^{3}$ Universidad Centroccidental Lisandro Alvarado, Cabudare, Venezuela, mparra@ucla.edu.ve

${ }^{4}$ Universidad Centroccidental Lisandro Alvarado, Cabudare, Venezuela, cgomez@ucla.edu.ve

${ }^{5}$ Universidad Centroccidental Lisandro Alvarado, Cabudare, Venezuela, mariselaestanga@ucla.edu.ve

${ }^{6}$ Universidad Centroccidental Lisandro Alvarado, Cabudare, Venezuela, yelitzagarcia@ucla.edu.ve

Cómo citar / How to cite

B. Mendoza-Escalona; D. Torres-Rodríguez; L. M. Marcó; C. Gómez; M. Estanga-Barrios; Y. García-Orellana, "Concentración de metales pesados en suelos agrícolas bajo diferentes sistemas de labranza", TecnoLógicas, vol. 24, nro. 51, e1738, 2021.

https://doi.org/10.22430/22565337.1738 


\section{Resumen}

Los metales pesados son elementos minerales cuya acumulación en el suelo, el agua y los tejidos vegetales constituyen un riesgo para la salud pública. Enfermedades cancerígenas, renales y hepáticas están asociadas a altas concentraciones de estos elementos. De igual forma, la acumulación de metales pesados está relacionada con actividades industriales, pero también a la producción agrícola, producto del uso excesivo de agroquímicos. Para evaluar los riesgos de contaminación por metales pesados en suelos agrícolas de la localidad de Turén al noroccidente de Venezuela, se midió el contenido de $\mathrm{Cd}$, $\mathrm{Cu}, \mathrm{Co}, \mathrm{Zn}, \mathrm{Fe}, \mathrm{Mn}, \mathrm{Ni}$, Ar y Se en suelos bajo dos sistemas de labranza - convencional y siembra directa - en dos posiciones fisiográficas: napa alta y napa baja, mediante fluorescencia de rayos X. Las muestras fueron tomadas a dos profundidades: 0-10 y 10-20 cm, con 9 muestras por posición dentro de cada sistema de labranza. Los resultados mostraron que el contenido de $\mathrm{Co}, \mathrm{Cu}$, $\mathrm{Ni}$, Cd y Zn fue mayor en el sistema de siembra directa debido al uso prolongado de abonos fosfatados Asimismo, la mayor acumulación se observó en la napa baja, lo cual estuvo relacionado a un mayor contenido de arcilla y a la capacidad de intercambio catiónico. Finalmente, se determinó que los valores de $\mathrm{Co}, \mathrm{Cu}$ y $\mathrm{Cd}$ estuvieron por encima de los valores máximos permitidos por las normas ambientales internacionales La acumulación de metales pesados en suelos de Turén, tanto en el sistema de labranza convencional como en el de siembra directa, son producto del uso continuo de agroquímicos, en particular de la fertilización fosfatada, por lo que se recomienda reducir el uso de agroquímicos, además de llevar cabo acciones de biorremediación para su eliminación del suelo y evitar los problemas de contaminación que a largo plazo y problemas de salud asociada a la presencia de los mismos en suelos y aguas.

\section{Palabras Claves}

Contaminación del suelo, deterioro ambiental, suelos agrícolas, medidas de suelo.

\section{Abstract}

Heavy metals are mineral elements whose accumulation in soils, water, and vegetal tissues constitutes a public health risk. High concentrations of these elements are associated with cancer and kidney and liver diseases. The accumulation of such metals is the consequence of industrial activities, but also agricultural production due to the excessive use of agrochemicals. Therefore, this study assessed the heavy metals contamination risk in Turen, in the northwest of Venezuela. $\mathrm{Cd}, \mathrm{Cu}, \mathrm{Co}, \mathrm{Zn}, \mathrm{Fe}, \mathrm{Mn}, \mathrm{Ni}, \mathrm{As}$, and Se were found in soils under two tillage systems (conventional and direct sowing) at two physiographic positions: high and low napa by $\mathrm{X}$ - ray fluorescence. The samples were taken at two depths, i.e., $0-10$ and $10-20 \mathrm{~cm}$, with nine samples per position in each tillage system. The results show that the content of $\mathrm{Co}, \mathrm{Cu}, \mathrm{Ni}, \mathrm{Cd}$, and $\mathrm{Zn}$ was higher in direct sowing due to the extended use of phosphate fertilizers. The greatest accumulation was observed at the low napa, which is associated with higher clay content and cation exchange capacity. The values of $\mathrm{Co}, \mathrm{Cu}$, and $\mathrm{Cd}$ were higher than the maximum allowable levels in most international environmental regulations. The accumulation of heavy metals in Turén soils, both in the conventional tillage system and in the direct sowing system, are the result of the continuous use of agrochemicals, in particular phosphate fertilization, so it is recommended to reduce the use of agrochemicals, in addition to carrying out bioremediation actions to eliminate them from the soil and avoid long-term contamination problems and health problems associated with their presence in soils and waters.

\section{Keywords}

Soil contamination, environmental impact, agricultural soils, soil measurements. 


\section{INTRODUCCIÓN}

Los metales pesados constituyen un riesgo potencial para la salud pública, así como para la biodiversidad vegetal y animal. Se ha reportado que la presencia de metales pesados está asociado a enfermedades como cáncer, daños hepáticos y renales [1], [2]. Los riesgos de contaminación de suelos por metales pesados generalmente se relacionan con actividades industriales como la minería [3], [4] y la explotación petrolera, siendo las fuentes potenciales de contaminación las aguas residuales, los lodos de cervecería y papelería, así como, los compost no tratados, entre otros [5], [6], [7], por lo que la probabilidad de encontrar suelos contaminados es más factible en zonas urbanas y periurbanas que en zonas agrícolas [8].

Las actividades agrícolas también pueden contaminar el suelo a largo plazo, debido al uso de fertilizantes y agroquímicos, en cuya constitución se encuentran metales pesados como el cobre [9], [10]; asimismo el uso prolongado de compost y aguas residuales sin tratar pueden conllevar al incremento de los niveles de metales pesados en el suelo, arrojando valores superiores a los permitidos en la legislación nacional e internacional.

La acumulación de metales pesados en el suelo se potencia por las interacciones que allí ocurren, como la formación de complejos con compuestos orgánicos o la adsorción en la micela coloidal. En principio estas propiedades le permitirían actuar al suelo como filtro ambiental; sin embargo, las actividades agrícolas que incluyan cambios en el pH pueden hacer disponibles estos metales, bajo el riesgo que estos sean traslocados a la planta [11], al igual que en suelos arenosos, donde existe la posibilidad de que los mismo se lixivien, contaminando las aguas subterráneas [12], [13].

La evaluación de los niveles de metales pesados es una obligación para los productores agrícolas y los agrotécnicos, dado que hoy en día los parámetros de calidad exigen productos libres de contaminantes, con el fin de garantizar a los consumidores un producto con calidad e inocuidad.

Dado esto, y considerando que la zona agrícola de Turén se ha caracterizado durante más de 30 años por el uso excesivo de agroquímicos, lo cual potencia el riesgo de contaminación por metales pesados, el objetivo de esta investigación fue evaluar la concentración de metales pesados en suelos y los riesgos de fitotoxicidad asociado a la presencia de los mismos.

\section{MATERIALES Y MÉTODOS}

\subsection{Materiales}

La investigación se realizó en suelos agrícolas de la localidad de Turén bajo diferentes sistemas de labranza. Las características y ubicación del suelo, así como los sistemas de labranza usado, se describen a continuación:

\subsubsection{Ubicación y características del sitio de estudio}

El estudio tuvo lugar en un suelo Fluventic Haplusteps, limoso, fino, mixto calcáreo isohipertérmico, bajo dos sistemas de labranza: labranza convencional (LC) y labranza cero o siembra directa (SD), ubicado en la localidad de Turén, al noroccidente de Venezuela, coordenadas entre $9^{\circ} 00^{\prime} 16^{\prime \prime}$ y $9^{\circ} 29^{\prime} 04^{\prime \prime}$ de latitud norte y los $68^{\circ} 39^{\prime} 50^{\prime \prime}$ y $69^{\circ} 12^{\prime} 17^{\prime \prime}$ de longitud oeste, con altura sobre el nivel del mar de $160 \mathrm{~m}$, precipitación anual promedio de $1424 \mathrm{~mm}$ y una temperatura media anual de $27^{\circ} \mathrm{C}$. El suelo bajo estudio, se encuentra 
ubicado en la planicie aluvial de desborde del río, con poca pendiente general (inferior al $0,3 \%)$ y topografía regular y plana. Se seleccionaron dos sitios de muestreo en cada sistema de manejo en función de su posición fisiográfica, tomando como referencia la parte alta y baja de la napa de limo de desborde sobre la cual se identificó el suelo. A continuación, se describe el manejo de los dos sistemas (Figura 1).

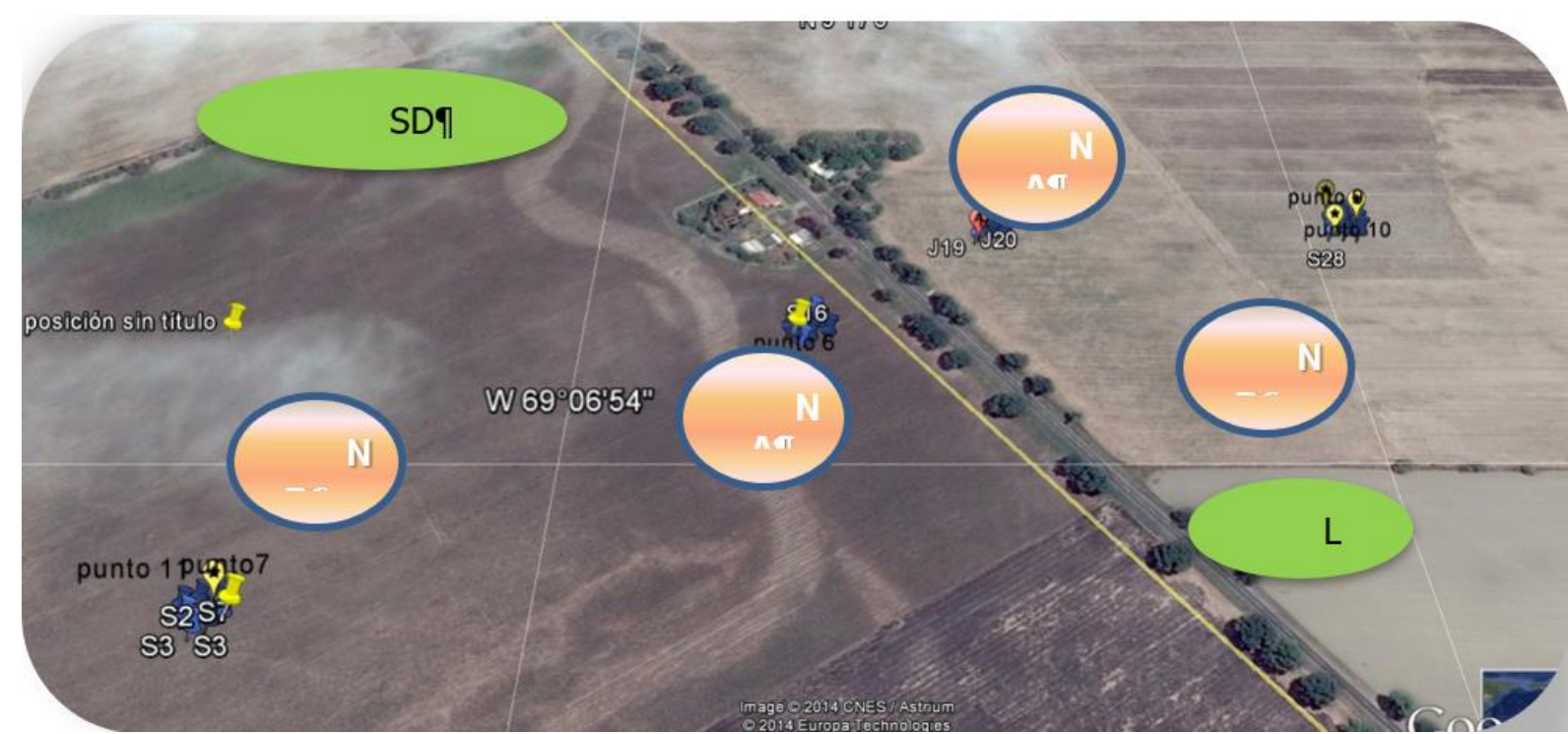

Figura 1. Descripción de los sistemas de manejo agrícola en dos predios de la Colonia Agrícola de Turén. Fuente: elaboración propia.

\subsubsection{Siembra directa}

Considerando las características descritas anteriormente del suelo y su ubicación, el manejo de este sistema consistió en diez años sin labranza donde se sembró bajo un sistema de rotación maíz (Zea mays L), girasol (Helianthus annuus) y maíz-leguminosa, para el control de malezas se usó un herbicida preemergente: glifosato, $2 \mathrm{~L} \mathrm{ha}^{-1}$ con 2,4-D 500 cc ha1.

Posemergente: atrazina, $1 \mathrm{~kg} \mathrm{ha-1,} \mathrm{con} \mathrm{dos} \mathrm{aplicaciones,} \mathrm{la} \mathrm{fertilización} \mathrm{durante} \mathrm{la}$ siembra fue usando el fertilizante compuesto (10-20-20), en dosis de $300 \mathrm{~kg} \mathrm{ha}^{-1}$ y urea: 100 $\mathrm{kg} \mathrm{ha}^{-1}$ como reabono en dosis de $150 \mathrm{~kg} \mathrm{ha}^{-1}$ de urea, la cual fue aplicada 25 días después de la siembra; asi como la aplicación de $50 \mathrm{~kg} \mathrm{ha}^{-1} \mathrm{de} \mathrm{KCl}$, vía foliar. La Semilla usada fue tratada con thiodicarb + imidacloprid, se realizó Quema de residuos de cosecha de maíz, mientras que los residuos de girasol permanecen en la superficie. Siembra de maíz en junio. La Densidad de siembra usada fue de 80,000 semillas ha-1.

\subsubsection{Labranza convencional}

Se hizo mecanización con tres o dos pases de rastra (profundidad $10-15 \mathrm{~cm}$ ), el sistema de siembra fue de rotación maíz-girasol, el control de malezas fue químico, usando el Herbicida preemergente: Pendimethalin, $3 \mathrm{~L}$ ha- ${ }^{-1}$ Posemergente: atrazina, $1 \mathrm{~kg} \mathrm{ha-}{ }^{-1}$ La Fertilización durante la siembra se hizo aplicando el fertilizante compuesto (10-20-20) en dosis $300 \mathrm{~kg} \mathrm{ha-1,} \mathrm{y} 15$ días después de la siembra se hizo un reabono con urea a razón de $150 \mathrm{~kg} \mathrm{ha-1}$, la semilla no recibió pretratamiento, se realizó Quema de residuos de cosecha de maíz y girasol, la siembra de maíz fue en junio con una densidad de siembra: 80000 semillas ha- ${ }^{1}$. 


\subsection{Métodos}

Para llevar a cabo la investigación, los procedimientos consistieron en muestreo de suelo, evaluación de los atributos químicos del mismo y análisis estadísticos. Los métodos usados son descritos a continuación:

\subsubsection{Diseño muestreo de suelo}

Se realizó un muestreo en la napa alta (NA) y en la napa baja (NB). De cada sistema de manejo se delimitó un área de $900 \mathrm{~m}^{2}$ que se dividió en cuadrículas de $3 \mathrm{x} 3$ puntos, distanciados a $10 \mathrm{~m}$ entre sí, para un total de 9 puntos de muestreo. El muestreo de suelo se realizó antes de la siembra de maíz. En cada punto se tomaron muestras disturbadas de suelo a dos profundidades $(0-10$ y $10-20 \mathrm{~cm})$, con tres repeticiones. Los análisis se realizaron en el Laboratorio de Suelos de la Unidad de Investigación de Suelos y Nutrición Mineral de Plantas del decanato de Agronomía de la UCLA (UISNMP) y la Unidad de Análisis Instrumental.

\subsubsection{Atributos químicos evaluados}

A las muestras de suelo, secadas y tamizadas $(2 \mathrm{~mm})$, se les determinó el contenido de arcilla, limo y arena por método de Bouyoucos [14], los mismo que el del carbono orgánico [15]; el pH, mediante determinación potenciométrica en relación suelo - agua 1:2,5; la conductividad eléctrica (CE), usando conductímetro en relación suelo- agua 1:2,5; la capacidad de intercambio (CIC), por extracción con cloruro de amonio y cuantificación por absorción atómica; la capacidad de intercambio catiónico (CIC), se realizó siguiendo los métodos descritos por [16].

Para el determinar el contenido de $\mathrm{Cd}, \mathrm{Cu}, \mathrm{Co}, \mathrm{Zn}, \mathrm{Fe}, \mathrm{Mn}, \mathrm{Ni}, \mathrm{Ar}$ y Se en suelos bajo dos sistemas de labranza (convencional y siembra directa) en dos posiciones fisiográficas (napa alta y napa baja), se tomaron 2 gramos de suelos, los cuales fueron previamente pesados y secados al aire por 24 horas; una vez seca, las muestras fueron molidas usando un mortero de ágata, donde posteriormente se colocaron en un portamuestra plástico; asimismo, para la florescencia de rayo X se usó una pistola de fluorescencia Bruker Tracer III-V PXRF (aka "gun"); para la cuantificación de los elementos, se usaron los programas (Bruker X-rayOps, S1PXRF 3.8.30 and Spectra 7.2.1.1).

\subsubsection{Análisis estadístico}

Según el muestreo realizado, el análisis estadístico consistió en realizar un estudio de varianza mediante comparación de las posiciones fisiográficas y profundidades en cada sistema de manejo por separado. El modelo estadístico aplicado se corresponde con un experimento factorial con dos factores: posición fisiográfica (NA y NB) y profundidad (0-10 y 10-20 cm). Para cada combinación de posición y profundidad se tomaron 9 muestras.

En los casos necesarios, se realizaron comparaciones de medias por la prueba de la diferencia honestamente significativa (DHS) de Tukey $(p<0,05)$; asimismo se determinaron estadísticas básicas como la media y la desviación estándar. Los análisis estadísticos se realizaron usando el paquete estadístico InfoStat versión 1.1 (2002). 


\section{RESULTADOS Y DISCUSIÓN}

El suelo bajo estudio fue un Fluventic Haplustep, el cual fue descrito en una investigación preliminar [17], el mismo se caracterizó por presentar texturas francas a arcillosas (Tabla 1) y valores medianos de contenido de materia orgánica y de capacidad de intercambio catiónico.

Tabla 1. Distribución de tamaño de partículas de un suelo bajo dos sistemas de labranza en dos posiciones fisiográficas a dos profundidades. Fuente: elaboración propia.

\begin{tabular}{ccccccccc} 
& \multicolumn{4}{c}{ Siembra directa } & \multicolumn{3}{c}{ Labranza convencional } \\
\hline & \multicolumn{2}{c}{ Napa alta } & \multicolumn{2}{c}{ Napa baja } & \multicolumn{2}{c}{ Napa alta } & \multicolumn{2}{c}{ Napa baja } \\
\hline $\begin{array}{c}\text { Profundidad } \\
\text { cm }\end{array}$ & $0-10$ & $10-20$ & $0-10$ & $10-20$ & $0-10$ & $10-20$ & $0-10$ & $10-20$ \\
\hline Arena \% & 47 & 49 & 22 & 27 & 31 & 32 & 9 & 9 \\
Limo \% & 38 & 36 & 56 & 52 & 53 & 49 & 59 & 59 \\
Arcilla \% & 15 & 15 & 22 & 21 & 16 & 19 & 32 & 32 \\
Textura & F & F & FL & FL & FL & F-FL & FAL & FAL \\
CIC cmol(+) & 10,7 & 9,23 & 11,72 & 11,03 & 9,93 & 10,42 & 12,91 & 12,74 \\
Kg-1 & 19,93 & 11,1 & 25,7 & 12 & 24,3 & 15,9 & 24,4 & 16,3 \\
MO g Kg-1 & 19,4
\end{tabular}

F: franco; FL: franco limoso; FAL: franco arcillo limoso

En la Tabla 2 se muestra el contenido de metales pesados en el suelo, bajo dos posiciones fisiográficas en dos sistemas de labranza.

Tabla 2. Contenido de metales pesados bajo dos sistemas de labranza en dos posiciones fisiográficas a dos profundidades. Fuente: elaboración propia.

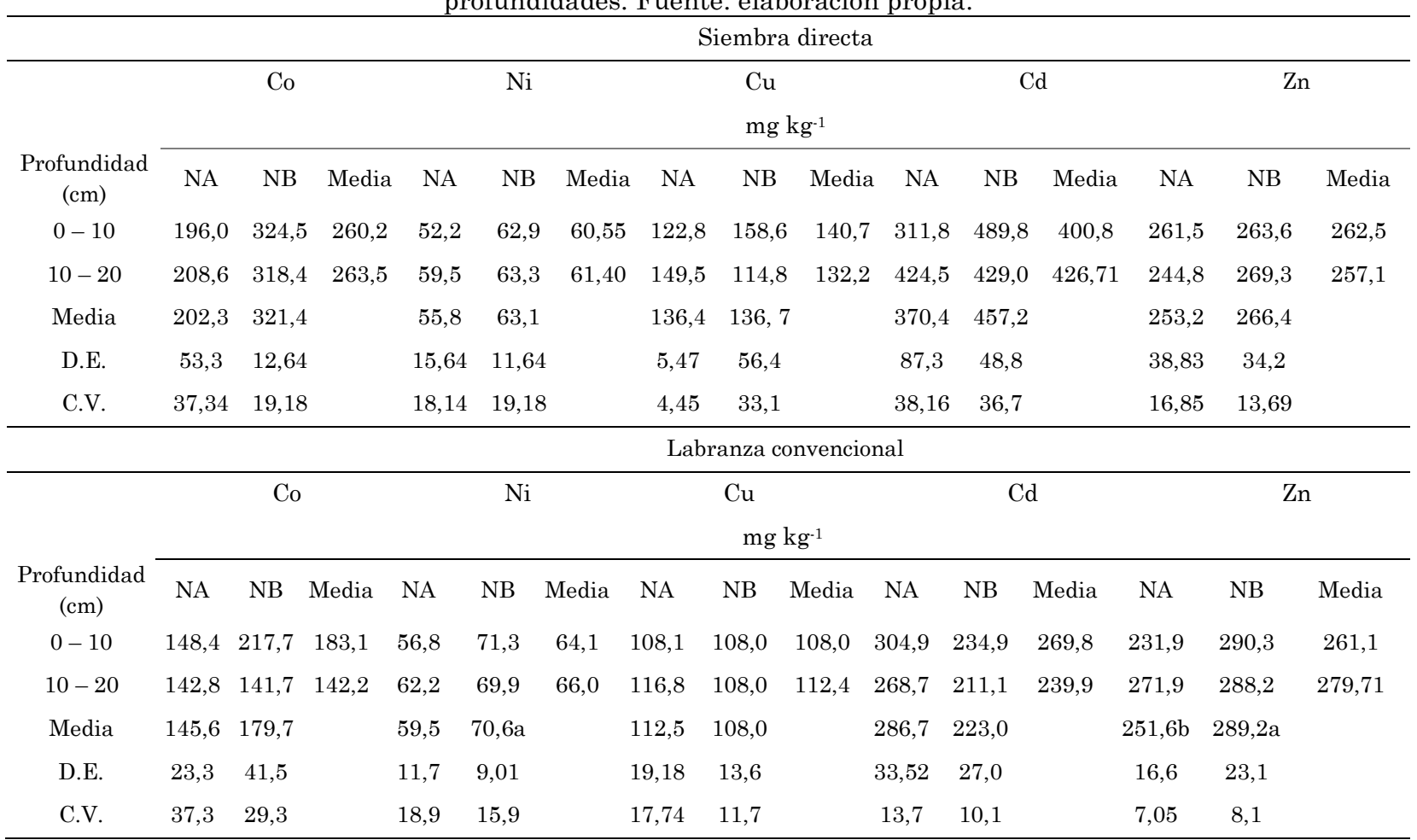

NA: napa alta; NB: napa baja. 
Para los dos sistemas de labranza evaluados, no se observaron diferencias significativas $(\mathrm{P}<0,05)$ al comparar el contenido de metales pesados entre profundidad. Sin embargo, se encontraron diferencias significativas debido a la posición fisiográfica para $\mathrm{Ni}$ y Zn en el suelo bajo labranza convencional, no así para el resto de los metales pesados.Los metales pesados fueron determinados por fluorescencia de rayos $\mathrm{X}$, el cual es un método más rápido que el de la espectroscopia de absorción atómica, y no existe subestimación de las concentraciones de metales pesados, que ocurre por la interacción entre los metales y la materia orgánica del suelo y los colides del mismo que impiden una extracción completa de estos.

Los resultados indican que las concentraciones Ni y Zn fueron afectadas por los cambios texturales debido a la posición en el paisaje, generando acumulación en las posiciones más bajas debido a la escorrentía.

En ambos sistemas de labranza se observó una tendencia a la acumulación de metales pesados en la napa baja, encontrándose que la acumulación de arcilla está relacionada al incremento de metales pesados en el suelo, debido a que estas poseen cargas negativas en su superficie o en el espacio interlaminar que confiere a los suelos la capacidad de retener elementos [18], lo cual esta correlacionado con la capacidad de intercambio catiónico (CIC).

En general, a mayor CIC, mayor es la cantidad de ion adsorbido [19], [20], por lo que el mayor contenido de metales pesados fue encontrado en la napa baja.

Asimismo, los minerales del suelo están formados por óxidos como los de titanio, silicio, hierro, manganeso y aluminio, siendo los tres últimos los más importantes en la retención de metales pesados [21], [22], [23]. Los más abundantes son los óxidos de hierro y pueden adsorber metales traza como $\mathrm{Co}, \mathrm{Cr}, \mathrm{Mn}, \mathrm{Mo}, \mathrm{Ni}, \mathrm{V}$ y $\mathrm{Zn}$. En el suelo estudiado predominaron los óxidos de silicio, titanio, hierro y manganeso, lo que favoreció la adsorción de los metales pesados (Figura 2).

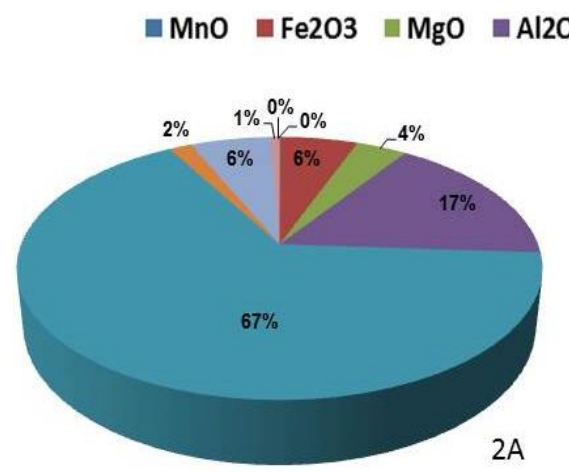

$\llbracket \mathrm{SiO} 2 \backsim \mathrm{K} 2 \mathrm{O} \backsim \mathrm{CaO} \backsim \mathrm{TiO} 2 \backsim \mathrm{V}$
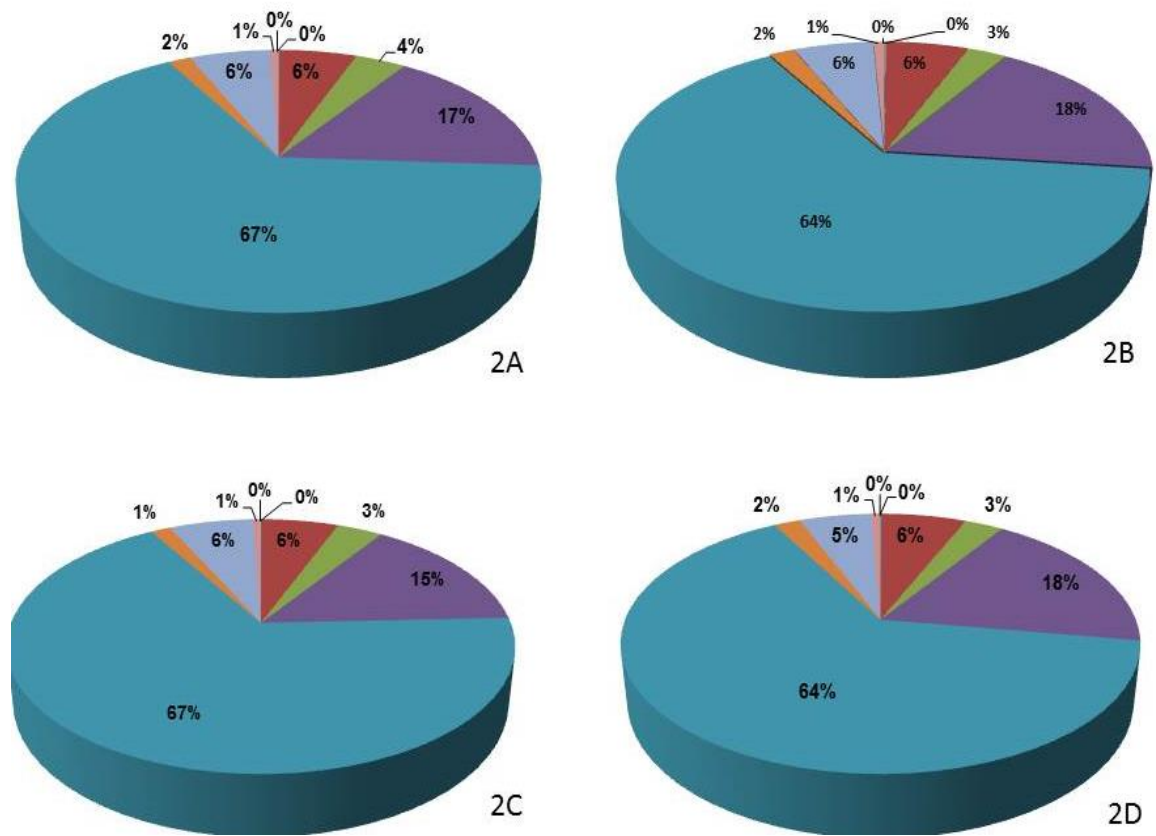

Figura 2. Composición mineral del suelo bajo: siembra directa (A. napa alta y B. napa baja) y labranza convencional (C. napa alta y D. napa baja), respectivamente, en la Colonia Agrícola de Turén.

Fuente: elaboración propia. 
La acumulación de metales en el suelo no solamente fue afectada por los factores edáficos como el contenido de arcilla y la CIC, sino que el sistema de manejo influyó en el contenido de metales pesados; en tal sentido se observó, en el caso del cobalto y el cadmio, que el contenido de metales pesados fue mayor en los sistemas de labranza bajo siembra directa en comparación con los sistemas bajo labranza convencional. Aunque, a largo plazo, los sistemas de siembra directa mejoran las propiedades físicas del suelo, debido a que se requiere mayor aplicación de agroquímicos, los cuales en su composición poseen residuos de metales pesados.

En tal sentido, los suelos donde a lo largo de muchos años se han aplicado fertilizantes fosfatados, poseen mayor riesgo de acumulación de metales pesados [24], [25]. Esto se debe a la presencia de impurezas de la materia prima (roca fosfórica). Por otra parte, los metales pesados, que se encuentran en concentraciones variables, persisten en los fertilizantes comerciales. De tal forma, los fertilizantes fosfatados son portadores de cadmio, cinc, cobalto, cobre, cromo, flúor, níquel, plomo y muchos otros elementos químicos, incluyendo componentes radioactivos [26], [27]. Estos elementos no sólo se acumulan en los suelos, sino que, dado que algunos como el cadmio poseen formas solubles en los fertilizantes, su utilización durante largos períodos de tiempo también causa una mayor contaminación.

Ahora bien, para evaluar los riesgos de contaminación por metales pesados, se compararon los valores obtenidos con concentraciones de metales pesados totales de referencia esperados en suelos agrícolas y con límites máximos permitidos según la legislación ambiental de Chile [3], Canadá [28] y la Unión Europea (Tabla 3), encontrándose que los valores de cobalto superan la norma europea; los de níquel y cobre, la norma canadiense; y los de cadmio y zinc superan todas las legislaciones ambientales reportadas en esta investigación.

Tabla 3. Comparación del contenido de metales pesados totales en suelo bajo siembra directa (SD) y labranza convencional (LC) con valores de referencia y límites máximos permitidos (LMP)

Fuente: elaboración propia.

\begin{tabular}{|c|c|c|c|c|c|c|c|c|c|c|}
\hline & \multicolumn{2}{|c|}{ Co mg kg-1 } & \multicolumn{2}{|c|}{ Ni mg kg-1 } & \multicolumn{2}{|c|}{ Cu mg kg-1 } & \multicolumn{2}{|c|}{ Cd mg kg-1 } & \multicolumn{2}{|c|}{ Zn mg kg-1 } \\
\hline & NA & NB & NA & NB & NA & NB & NA & NB & NA & NB \\
\hline SD & 321,44 & 202,27 & 63,13 & 58,83 & 136,88 & 136,17 & 457,15 & 370,36 & 266,43 & 253,15 \\
\hline LC & 145,60 & 179,67 & 59,45 & 70,61 & 112,46 & 107,99 & 286,71 & 223,00 & 251,55 & 289,23 \\
\hline LMP Ecuador & \multicolumn{2}{|c|}{--} & \multicolumn{2}{|c|}{--} & \multicolumn{2}{|c|}{--} & \multicolumn{2}{|c|}{2} & \multicolumn{2}{|c|}{200} \\
\hline LMP Holanda & \multicolumn{2}{|c|}{$\cdots$} & \multicolumn{2}{|c|}{--} & \multicolumn{2}{|c|}{$\cdots$} & \multicolumn{2}{|c|}{0,8} & \multicolumn{2}{|c|}{140} \\
\hline LMP Chile & \multicolumn{2}{|c|}{$\ldots$} & \multicolumn{2}{|c|}{112} & \multicolumn{2}{|c|}{150} & \multicolumn{2}{|c|}{2} & \multicolumn{2}{|c|}{175} \\
\hline LMP Canadá & \multicolumn{2}{|c|}{$\cdots$} & \multicolumn{2}{|c|}{32} & \multicolumn{2}{|c|}{100} & \multicolumn{2}{|c|}{1,6} & \multicolumn{2}{|c|}{220} \\
\hline $\begin{array}{l}\text { LMP en suelos de } \\
\text { Europa }\end{array}$ & \multicolumn{2}{|c|}{50} & \multicolumn{2}{|c|}{100} & \multicolumn{2}{|c|}{100} & \multicolumn{2}{|c|}{5} & \multicolumn{2}{|c|}{300} \\
\hline $\begin{array}{c}\text { Intervalo en } \\
\text { suelos }\end{array}$ & \multicolumn{2}{|c|}{$\cdots$} & \multicolumn{2}{|c|}{$10-100^{* *}$} & \multicolumn{2}{|c|}{$2-100 * *$} & \multicolumn{2}{|c|}{$0,01-7 * *$} & \multicolumn{2}{|c|}{$10-300^{* *}$} \\
\hline LMP en suelo & \multicolumn{2}{|c|}{--} & \multicolumn{2}{|c|}{30} & \multicolumn{2}{|c|}{50} & & & & \\
\hline $\begin{array}{l}\text { LMP en suelos } \\
\text { con } p H>7\end{array}$ & & & & & & & & & & \\
\hline
\end{tabular}

NA: napa alta; NB: napa baja.

Los resultados indican que hay que prestar atención a los contenidos de $\mathrm{Co}, \mathrm{Cu}$ y $\mathrm{Cd}$ en ambos sistemas de manejo, especialmente en $\mathrm{SD}$, porque el $\mathrm{Cu}$ se encuentra por encima de algunos de los niveles de referencia permitidos en suelo, mientras que el Co y el Cd superan significativamente en todos los casos el límite máximo permisible. Los niveles de cobalto superaron los $200 \mathrm{mg} \mathrm{kg}^{-1}$, lo cual está por encima del máximo permitido en diversas normas 
ambientales. Algunos autores sugieren que cuando el cobalto supera los $100 \mathrm{mg} \mathrm{kg}$-1 en el suelo se deben realizar tratamientos de remediación, lo cual incluye el uso de plantas y microrganismos. Aunque la concentración de cobalto está en niveles superiores a los permitidos, los riesgos de movilidad y translocación a las plantas son bajos. Se ha reportado que este metal es fuertemente adsorbido por los óxidos de hierro y manganeso, así como los carbonatos, además que forma quelatos con la materia orgánica [29] [30]. De igual forma, en $\mathrm{pH}$ alcalinos la movilidad de este elemento resulta muy baja [31].

En el caso del cadmio (Tabla 4), las fuentes de contaminación están asociadas a las actividades industriales: minería, aplicación de enmiendas para mejorar las propiedades físicas y químicas del suelo y el uso de fertilizantes fosfatados [32] [33]. Este último aspecto es fundamental en la zona bajo estudio, dado que se ha reportado [34] que, en los llanos venezolanos, donde se encuentra la localidad de Turén, se aplican cantidades excesivas de fertilizantes fosfatados. La distribución y disponibilidad de los metales pesados en el perfil del suelo está determinada por las características físicas, químicas y biológicas de este sistema, que influyen sobre la actividad y concentración de los metales en la solución del suelo y, por ende, afectan su disponibilidad. Entre estas variables se destacan el contenido y tipo de arcillas y la CIC [35].

Tabla 4. Contenido promedio de cadmio disponible en suelos bajo dos sistemas de labranza en dos posiciones fisiográficas a dos profundidades. Fuente: elaboración propia.

\begin{tabular}{ccccc}
\hline & \multicolumn{2}{c}{ Siembra directa } & \multicolumn{2}{c}{ Labranza convencional } \\
\hline Profundidad $\mathbf{( c m})$ & & Cadmio disponible $\left(\mathrm{mg} \mathrm{kg}^{-1}\right)$ & Napa baja \\
\hline & Napa alta & Napa baja & Napa alta & 32,99 \\
$\mathbf{0 - 1 0}$ & 12,82 & 16,64 & 36,38 & 32,19 \\
$\mathbf{1 0 - 2 0}$ & 15,76 & 10,50 & 36,08 & 37,98 \\
\hline
\end{tabular}

En suelos con alta capacidad de intercambio catiónico y alto contenido de arcilla se espera que la disponibilidad de cadmio sea baja, ya que el mismo quedará adsorbido en las partículas de arcillas, evitando que sea tomado por las plantas o pase a las aguas subterráneas [33]. Del mismo modo, en suelos de Mariara, al centro de Venezuela, con características físicas y químicas similares a la de la presente investigación (contenido mediano de materia orgánica, textura arcillosa y alta CIC), se presentó una alta capacidad de adsorción de cadmio.

Sin embargo, en los suelos estudiados, la concentración de cadmio disponible es muy elevada, con valores superiores a $30 \mathrm{mg} \mathrm{kg}^{-1}$ en el sistema de labranza convencional, mientras que en los sistemas de siembra directa este valor se reduce por debajo de $18 \mathrm{mg} \mathrm{kg}$ 1, el cual es similar a lo reportado por [36]. En la colonia agrícola de Turén se aplican cantidades excesivas de fertilizante fosfatado, lo que está asociado a concentración de cadmio elevada.

El caso de la contaminación por cobre en zonas agrícolas venezolanas es debido a la aplicación constante de agroquímicos que usan al cobre como su elemento principal. Estos resultados coinciden con los reportados en otras regiones latinoamericanas, donde las malas prácticas en el uso de agroquímicos han provocado serios problemas de contaminación. [37] reportaron que en varias zonas de Costa Rica se encontraron contenidos de cobre en la capa arable de hasta de $2000 \mathrm{mg} \mathrm{kg}^{-1}$. El promedio de cobre en los suelos naturales en el mundo se encuentra en el ámbito de 13 a $24 \mathrm{mg} \mathrm{kg}^{-1}$, mientras que en suelos contaminados por el metal se han reportado valores de hasta $4622 \mathrm{mg} \mathrm{kg}^{-1}$, en sitios cercanos a fundiciones. La 
absorción de cobre es básicamente independiente y está relacionada, en primer lugar, con los niveles de cobre disponible en el suelo. La disponibilidad de cobre para las plantas es menor en los suelos alcalinos y mayor en los suelos ácidos.

\section{CONCLUSIONES}

La acumulación de metales pesados en suelos de Turén, tanto en el sistema de labranza convencional como en el de siembra directa, son producto del uso continuo de agroquímicos, en particular de la fertilización fosfatada. Asimismo, la acumulación de metales pesados estuvo relacionada a una mayor acumulación de arcillas y CIC en las posiciones fisiográficas bajas, pues los valores más altos de metales pesados fueron encontrados en la napa baja, debido a un mayor contenido de arcilla y materia orgánica, aumentando la retención de nutrientes y el contenido de cationes.

Por otra parte, la mayor acumulación de zinc y níquel se asocia a mayores valores en contenido de arcilla y CIC en el suelo, lo cual mantiene a los metales pesados fuertemente adsorbidos en la micela coloidal.

De igual forma, las altas concentraciones de cobalto, níquel y cadmio estuvieron por encima de lo permitido en la mayoría de las normas internacionales, lo que representa un riesgo de fitotoxicidad para los cultivos sembrados en la zona, por lo que se recomienda tomar medidas de biorremediación para su eliminación del suelo y evitar los problemas de contaminación que a largo plazo contaminen las aguas y puedan afectar la salud de las poblaciones cercanas.

\section{AGRADECIMIENTOS}

Los autores agradecen al consejo de desarrollo humanístico y tecnológico de la universidad Lisandro Alvarado (CDCHT-UCLA) por el financiamiento parcial de la investigación a través de los fondos asignados en el programa de estímulo a la investigación Lisandro Alvarado (PEILA) en las ediciones 2019 y 2020 Colombia por su compromiso con incentivar el interés por la investigación científica.

\section{CONFLICTOS DE INTERÉS DE LOS AUTORES}

No hay conflicto de interés que declarar por parte de los autores.

\section{CONTRIBUCIÓN DE LOS AUTORES}

Betty Mendoza Escalona: conceptualización, diseño y desarrollo de la investigación y en la redacción y revisión final del manuscrito.

Duilio Torres-Rodríguez: desarrollo de la investigación, asesoramiento y revisión del manuscrito.

Lue Merú Marco: asesor en análisis químico instrumental.

Carlos Gómez: desarrollo de la investigación.

Marisela Estanga Barrios: desarrollo de la investigación. 
Yelitza García Orellana: desarrollo de la investigación y en la redacción y revisión final del manuscrito.

\section{REFERENCIAS}

[1] G. A. Engwa; P. U. Ferdinand; F. N. Nwalo; M. N. Unachukwu, "Mechanism and health effects of heavy metal toxicity in humans," In Poisoning in the Modern World-New Tricks for an Old Dog?. IntechOpen. 2019. https://doi.org/10.5772/intechopen.82511

[2] Y. Reyes; I. Vergara; O. Torres; M. Díaz Lagos; E. E. González Jimenez, “Contaminación por metales pesados: implicaciones en salud, ambiente y seguridad alimentaria," Rev.Ing., Investigación y Desarrollo, vol. 16, n. 2, pp. 66-77, Jul. 2016. URL

[3] Z. Martínez; M. González, "Contaminación de suelos agrícolas por metales pesados, zona minera El Alacrán, Colombia," Temas agrarios, vol. 22, no. 2, pp. 21-31. Jul. 2017. https://doi.org/10.21897/rta.v22i2.941

[4] I. Pérez; F. Martín, "Uso de parámetros indirectos para la evaluación de la contaminación de suelos por metales pesados en una zona minera de San Luis Potosí, México". Bol. Soc. Geol. Mex, vol. 67, no. 1, pp. 01-12. Apr. 2015. URL

[5] C. L. Hansen; D. Y. Cheong, "Chapter 26 - Agricultural Waste Management in Food Processing," en Handbook of Farm, Dairy and Food Machinery Engineering (Third Edition), Academic Press. pp. 673-716. 2019. https://doi.org/10.1016/B978-0-12-814803-7.00026-9

[6] Z. Chu; X. Fan; W. Wang; W. C Huang, "Quantitative evaluation of heavy metals' pollution hazards and estimation of heavy metals' environmental costs in leachate during food waste composting," Waste Management, vol. 84, pp. 119-128. Feb. 2019. https://doi.org/10.1016/j.wasman.2018.11.031

[7] W. Tian; Z. Zhang; X. Hu; R. Tian; J. Zhang; X. Xiao; Y. Xi, "Short-term changes in total heavy metal concentration and bacterial community composition after replicated and heavy application of pig manurebased compost in an organic vegetable production system," Biology and Fertility of Soils, vol. 51, no. 5, pp. 593-603. Mar. 2015. https://doi.org/10.1007/s00374-015-1005-4

[8] J. L. Cortés; F. Bautista; C. Delgado; P. Quintana; D. Aguilar; A. García; C. Figueroa; A. Gogichaishvili, "Spatial distribution of heavy metals in urban dust from Ensenada, Baja California, Mexico". Rev. Chapingo ser. cienc. for. ambient, vol. 23, no. 1, pp. 47-60, Apr. 2017. http://dx.doi.org/10.5154/r.rchscfa.2016.02.005

[9] E. Quinteros et al., "Heavy metals and pesticide exposure from agricultural activities and former agrochemical factory in a Salvadoran rural community," Environmental Science and Pollution Research, vol. 24, no. 2, pp. 1662-1676. Oct. 2016. https://doi.org/10.1007/s11356-016-7899-z

[10] Y. H. Senkondo; E. Semu; F. M. G. Tack, "Copper Bioavailability to Beans (Phaseolus vulgaris) in LongTerm Cu-Contaminated Soils, Uncontaminated Soils, and Recently Cu-Spiked Soils”, Soil and Sediment Contamination: An International Journal, vol. 24, no. 2, pp. 116-128, Jan. 2015. https://doi.org/10.1080/15320383.2014.920763

[11] J. D. Mahecha; J. M. Trujillo González; M. A. Torres Mora, "Contenido de metales pesados en suelos agrícolas de la región del Ariari, Departamento del Meta,” Orinoquia, vol. 19, no. 1, pp. 118-122. 2015. $\underline{\mathrm{URL}}$

[12] K. Page; M. J. Harbottle; P. J. Cleall; T. R. Hutchings, "Heavy metal leaching and environmental risk from the use of compost-like output as an energy crop growth substrate," Science of the Total Environment, vol. 487, pp. 260-271. Jul. 2014. https://doi.org/10.1016/j.scitotenv.2014.04.021

[13] E. F. Ledesma; A. Lozano-Lunar; J. Ayuso; A. P. Galvín; J. M. Fernández; J. R. Jiménez, "The role of pH on leaching of heavy metals and chlorides from electric arc furnace dust in cement-based mortars," Construction and Building Materials, vol. $183, \quad$ pp. $\quad 365-375 . \quad$ Sep. 2018. https://doi.org/10.1016/j.conbuildmat.2018.06.175

[14] G. J. Bouyoucos, "Directions for making mechanical analyses of soils by the hydrometer method," Soil Science, vol. 42, no. 3, pp. 225-230, Sep. 1936. https://doi.org/10.1097/00010694-193609000-00007

[15] A. Walkley; I. A. Black, "An examination of the Degtjareff method for determining soil organic matter, and a proposed modification of the chromic acid titration method," Soil science, vol. 37, no. 1, pp. 29-38. 1934. URL

[16] R. Gavlak; D. Horneck; R. Miller, "Soil, Plant and Water Reference Methods for the Western Region". 2da ed. WCC-103 Pub. Colorado State University, Fort Collins. EUA. pp. 37-47. 2005. URL

[17] B. Mendoza; E. Vera; A. Chassaigne; C. Gómez; D. Torres; Y. Bastidas, "Efecto de la posición fisiográfica y profundidad en dos sistemas de labranza sobre atributos de un suelo de Turén," Rev. Unell. Cienc. Tec., vol. 33, pp. 1-12. Apr. 2015. URL

[18] M. K. Uddin, "A review on the adsorption of heavy metals by clay minerals, with special focus on the past decade," Chemical Engineering Journal, vol. 308, pp. $438-462 . \quad 2017$. 
https://doi.org/10.1016/j.cej.2016.09.029

[19] L. Liu; X. Guo; C. Zhang; C. Luo; C. Xiao; R. Li, "Adsorption behaviours and mechanisms of heavy metal ions' impact on municipal waste composts with different degree of maturity," Environmental technology, vol. 40, no. 22, pp. 2962-2976. Apr. 2018. https://doi.org/10.1080/09593330.2018.1458908

[20] B. Huang et al., "Adsorption characteristics of $\mathrm{Cu}$ and $\mathrm{Zn}$ onto various size fractions of aggregates from red paddy soil," Journal of hazardous materials, vol. 264, pp. 176-183. Jan. 2014. https://doi.org/10.1016/j.jhazmat.2013.10.074

[21] A. Ullah; Y. Ma; J. Li; N. Tahir; B. Hussain, "Effective Amendments on Cadmium, Arsenic, Chromium and Lead Contaminated Paddy Soil for Rice Safety". Agronomy, vol. 10, no. 3, pp. 359. Mar. 2020. https://doi.org/10.3390/agronomy10030359

[22] P. N. Diagboya; B. I. Olu-Owolabi; K. O. Adebowale, "Effects of time, soil organic matter, and iron oxides on the relative retention and redistribution of lead, cadmium, and copper on soils," Environmental Science and Pollution Research, vol. 22, no. 13, pp. 10331-10339. Feb. 2015. https://doi.org/10.1007/s11356-015$\underline{4241-0}$

[23] F. Franco et al., "Low-cost aluminum and iron oxides supported on dioctahedral and trioctahedral smectites: A comparative study of the effectiveness on the heavy metal adsorption from water," Applied Clay Science, vol. 119, no. 2, pp. 321-332. Jan. 2016. https://doi.org/10.1016/j.clay.2015.10.035

[24] X. X. Chen; Y. M. Liu; Q. Y. Zhao; W. Qing Cao; X. Ping Chen; C. Q. Zou, "Health risk assessment associated with heavy metal accumulation in wheat after long-term phosphorus fertilizer application," Environmental Pollution, vol. 262, pp. 114348. Jul. 2020. https://doi.org/10.1016/j.envpol.2020.114348

[25] C. Su; L. Jiang; W. Zhang, "A review on heavy metal contamination in the soil worldwide: situation, impact and remediation techniques," Environmental Skeptics and Critics, vol. 3, no. 2, pp. 24-38. Jun. 2014. URL

[26] L. Zheng; B. Ying; Y. Tongming, "Effect of Cd on the Uptake and Translocation of Pb, Cu, Zn, and Ni in Potato and Wheat Grown in Sierozem," Soil and Sediment Contamination: An International Journal, vol. 28, no. 7, pp. 650-669. Jul. 2019. https://doi.org/10.1080/15320383.2019.1643289

[27] D. K. Gupta; S. Chatterjee; S. Datta; V. Veer; C. Wtalther, "Role of phosphate fertilizers in heavy metal uptake and detoxification of toxic metals," Chemosphere, vol. 108, pp. 134-144. Aug. 2014. https://doi.org/10.1016/j.chemosphere.2014.01.030

[28] J. Crossman; R. R. Hurley; M. Futter; L. Nizzetto, "Transfer and transport of microplastics from biosolids to agricultural soils and the wider environment," Science of The Total Environment, vol. 724, pp. 138334. Jul. 2020. https://doi.org/10.1016/j.scitotenv.2020.138334

[29] W. Chen et al., "Enhanced removal of lead ions from aqueous solution by iron oxide nanomaterials with cobalt and nickel doping," Journal of Cleaner Production, vol. 211, pp. 1250-1258. Feb. 2019. https://doi.org/10.1016/j.jclepro.2018.11.254

[30] B. Lange et al., "Copper and cobalt mobility in soil and accumulation in a metallophyte as influenced by experimental manipulation of soil chemical factors," Chemosphere, vol. 146, pp. 75-84. Mar. 2016. https://doi.org/10.1016/j.chemosphere.2015.11.105

[31] M. Jović; M. Šljivić-Ivanović; S. Dimović; J. Marković; I. Smičiklas, "Sorption and mobility of Co (II) in relation to soil properties," Geoderma, vol. 297, pp. 38-47, Jul. 2017. https://doi.org/10.1016/j.geoderma.2017.03.006

[32] M. A. Khan; S. Khan; A. Khan; M. Alam, "Soil contamination with cadmium, consequences and remediation using organic amendments," Science of the Total Environment, vol. 601- 602, pp. 1591-1605. Dec. 2017. https://doi.org/10.1016/j.scitotenv.2017.06.030

[33] N. Sánchez; C. Rivero; Y. Martínez, "Cadmio disponible en dos suelos de Venezuela: efecto del fósforo," Revista Ingeniería UC, vol. 18, no. 2, pp. 7-14. Ago. 2011. URL

[34] M. López; B. Rodríguez; M. España, "Tecnologías generadas por el Inia para contribuir al manejo integral de la fertilidad del suelo”. Agronomía Tropical, vol. 60, no. 4, pp. 315-330. Oct. 2010. URL

[35] W. Chai; Y. Huang; S. Su; G. Han; J. Liu; Y. Cao, "Adsorption behavior of Zn (II) onto natural minerals in wastewater. A comparative study of bentonite and kaolinite," Physicochemical Problems of Mineral Processing, vol. 53. no. 1. Pp. 264. 278, 2017. http://dx.doi.org/10.5277\%2Fppmp170122

[36] E. Vera; J. Viloria; R. Ramírez; R. Figueroa, "Análisis de brecha de rendimientos de maíz en parcelas comerciales”. Memorias XIX Congreso Venezolano de suelos. Calabozo-Guárico: CD ROM 1-5. URL

[37] R. Hernández; A. Alvarado; R. Romero, "Acumulación de cobre en plantas silvestres de zonas agrícolas contaminadas con el metal,” Rev. Ciencia y Tecnología, vol. 28, no. 1-2, pp. 55-61. Jul. 2012. URL 